\title{
OPTIMAL DESIGN OF RECONFIGURABLE FLOW LINES
}

\author{
GOYAL, K. K.; JAIN, P. K. \& JAIN, M.
}

Abstract: The present scenario is of ever increasing volatility in the global manufacturing environment and the fast paced evolution of manufacturing systems. The last decade has witnessed fast paced research and development in the field of Reconfigurable Manufacturing System (RMS). At the heart of RMS lies the reconfigurable machine tools (RMT) which are capable of performing multiple operations in their existing configuration and can further be reconfigured into other configurations to change the capacity and functionality. Thus a part can be processed by many feasible configurations of the RMT. In such an environment the configuration design of RMS plays a very crucial role in the efficient, cost effective and responsive functioning of the reconfigurable flow line. This chapter presents a methodology to design and optimize the reconfigurable flow lines by applying nondominated sorting genetic algorithm II considering multiple objectives i.e. cost, machine utilization, operational capability and machine reconfigurability. A case study has also been presented to demonstrate the design approach.

Key words: reconfigurable manufacturing system (RMS), reconfigurable machine tool (RMT), reconfigurable flow line, NSGA II
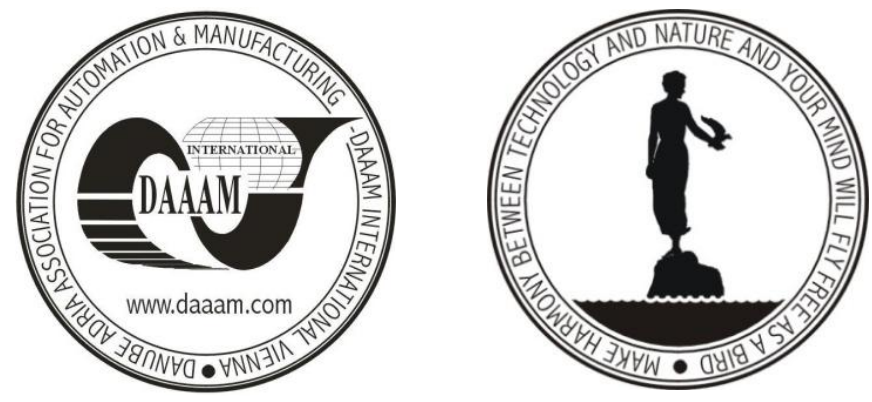

Authors' data: Research Scholar Goyal, K[apil] K[umar]*; Prof. Jain, P[ramod] K[umar]*; Dr. Jain, M[adhu]**, *Mechanical \& Industrial Engineering Department, IIT Roorkee, Roorkee, INDIA, **Department of Mathematics, IIT Roorkee, Roorkee, INDIA, kapilacad@gmail.com, pjainfme@iitr.ernet.in, madhufma@iitr.ernet.in

This Publication has to be referred as: Goyal, K[apil] K[umar]; Jain, P[ramod] K[umar] \& Jain, M[adhu] (2012). Optimally Designing the Reconfigurable Flow Lines, Chapter 13 in DAAAM International Scientific Book 2012, pp. 143-156, B. Katalinic (Ed.), Published by DAAAM International, ISBN 978-3-901509-86-5, ISSN 1726-9687, Vienna, Austria

DOI: $10.2507 /$ daaam.scibook.2012.13 


\section{Introduction}

The global economic competition, customization and rapid advancements in the product and process technologies have forced the manufacturers to inculcate the manufacturing responsiveness into their business model. The dramatically changing world in which, emerging economies, new ideas and philosophies of doing business, technical advancements and ever changing needs of customers is posing serious challenges to the survival of industries (Mehrabi et al., 2000). This change presents both a threat and an opportunity. To capitalize on the opportunity, an industry needs to possess manufacturing systems that can produce a wide range of products within its product/business portfolio cost effectively. Manufacturing systems have evolved over the years in response to an increasingly dynamic and global market with greater need for flexibility and responsiveness. Traditional Job Shops, Dedicated Manufacturing Systems (DMS), Cellular Manufacturing Systems (CMS) as well as Flexible Manufacturing Systems (FMS) all have their own difficulties and shortcomings to perform adequately and competitively in such a highly dynamic environment. However, fast paced advances in various supporting technologies have paved the way for a new manufacturing system paradigm named as Reconfigurable Manufacturing System (RMS) that meets the new manufacturing objectives with high responsiveness (Koren et al., 1999).

Liles and Huff (1990) have defined an RMS as a system capable of tailoring the configuration of manufacturing system to meet the production demand placed on it dynamically. The concept of 'modular manufacturing' defined by Tsukune et al. (1993) is also similar to the Reconfigurable Manufacturing System. Later in 1996 the Engineering Research Centre for Reconfigurable Manufacturing Systems (ERCRMS) was established at the University of Michigan, Ann Arbor to develop and implement reconfigurable manufacturing systems. Koren et al. (1999) defined RMS as:

"An RMS is designed at the outset for rapid change in its structure, as well as in hardware and software components, in order to quickly adjust the production capacity and functionality within a part family in response to sudden unpredictable market changes as well as introduction of new products or new process technology".

The most significant feature of the RMS is that the configuration of these systems evolves over a period of time in order to provide the functionality and capacity that is needed, and when it is needed (Mehrabi et al., 2000 ). Reconfigurable Machine Tool (RMT) lies at the heart of reconfigurable manufacturing system, which imparts RMS its distinguishing features i.e. customized functionalities and adjustable capacity through its changeable structure. The reconfigurable machine tools are modular machines comprising of different basic and auxiliary modules (Koren et al., 1999; Landers, 2000; Moon \& Kota, 2002). The basic modules are structural in nature like base, columns, and slide ways and the auxiliary modules are kinematical or motion providing modules such as spindle heads, tool changers, spacers, indexing units, adapter plates and angle structure, etc. The auxiliary modules are 
comparatively smaller, lighter and cheaper than the basic modules. Therefore they may be economically and rapidly changed with comparatively lesser effort. The RMTs can be rapidly reconfigured into many other configurations having different functionality and capacity by keeping its base modules and just changing the auxiliary modules. A machine is said to have multiple configurations if it can be converted into other configurations by just changing the auxiliary modules. Fig. 1 depicts the configuration of RMTs from the standard module library, in which first and second machine configuration (i.e. $m c_{1}^{1}$ and $m c_{1}^{2}$ ) of machine one and the first configuration of machine two (i.e. $m c_{2}^{1}$ ) are configured respectively by assembling the basic modules and auxiliary modules from the module library.

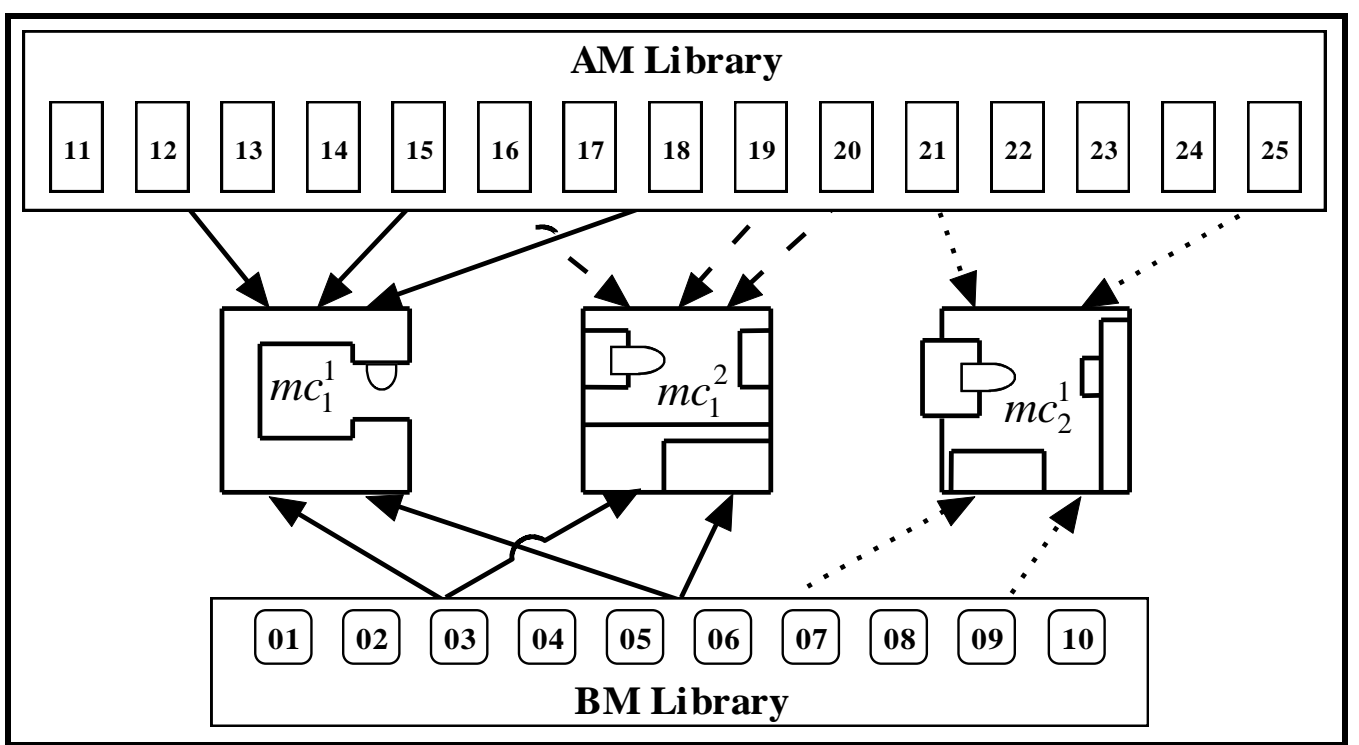

Fig. 1. Configuring RMT through module library adapted from Pattanaik et al., (2007)

The reconfigurability had been the key issue in reconfigurable manufacturing system which in general can be defined as the ability of a system to repeatedly change and reorient its components easily and cost effectively to achieve the variety of objectives. As shown in Fig. 2, the reconfigurability can be achieved at the system level by quickly adjusting the number of manufacturing lines/manufacturing cells. At the manufacturing line/manufacturing cell level reconfigurability can be achieved by adding/removing or changing the position of machines and material handling systems. The reconfigurability at machine level can be achieved by changing the functionality and capacity through adding/removing or readjusting the existing auxiliary modules. The RMS is built around a part family i.e. offering the exact capacity and functionality needed to process a part family and behaves as DMS during the production phase and can readily be reconfigured according to the new manufacturing requirements (Koren et al., 1999). Like DMS the most appropriate layout for the RMS is also the flow line layout to support mass manufacturing at the competitive cost. In most of the RMS modelling approaches flow line layout has been adopted (Dou et al., 2009; Youssef \& ElMaraghy 2006; Goyal et al., 2011b). Therefore authors have considered the flow line layout allowing paralleling of similar machines. 


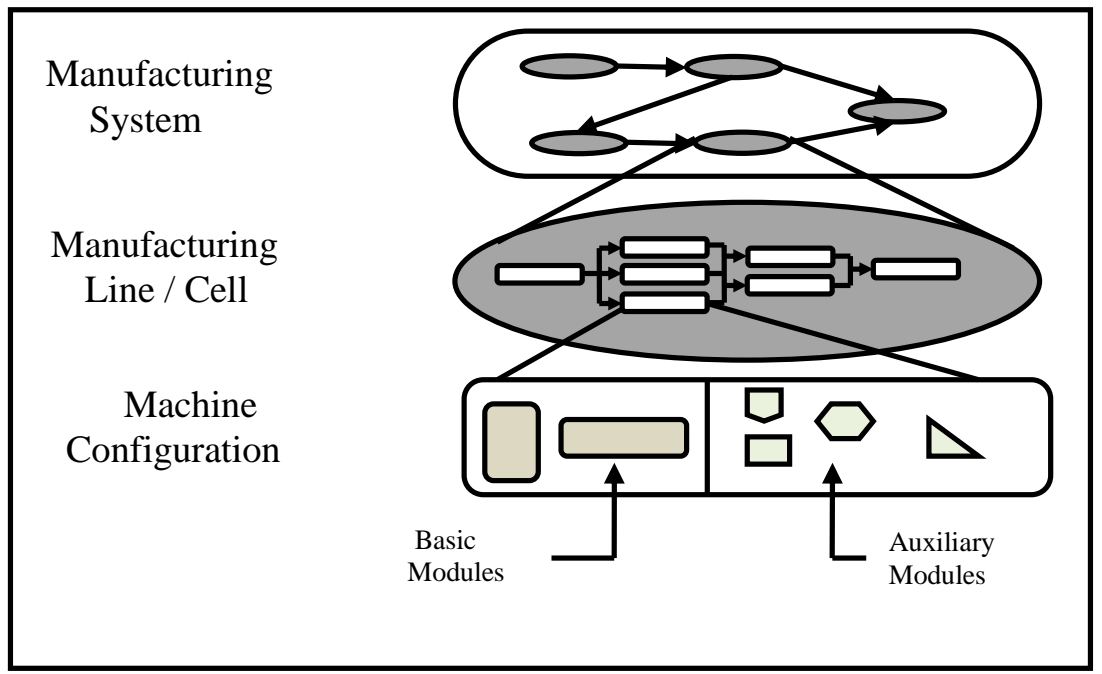

Fig. 2. The structure of a reconfigurable manufacturing system

Son (2000) and Youssef and ElMaraghy (2007) have modeled a multiple demand period configuration generation by first recording the $\mathrm{k}$ best configurations for all the demand periods based on cost as single performance criterion by applying Genetic Algorithm (GA) and in later stage selected the configurations based on configuration similarity and reconfiguration smoothness. Spicer and Carlo (2007) have attempted the multiple periods RMS modeling by considering the cost and reconfiguration as performance measures by using dynamic programming approach. But in their work only bases and spindles were considered as two RMT modules, which is far from realistic. Goyal et al. (2011a, 2011b) have developed the operational capability and machine reconfigurability to optimize the RMS configurations. Gumasta et al. (2011) have developed reconfigurability index for the system level changeability.

In nutshell the reconfigurable manufacturing systems offer several feasible alternative machine configurations for performing each operation and over the period demand along with the product mix changes which requires the change in configuration of the system. Therefore the problem of selecting the machines for various operations is very crucial and needs the elaborative performance measures so as to reduce the reconfigurations required at the later stage. In this chapter a novel approach is suggested for recording the non dominated solutions based on the multiple performance measures i.e. cost, machine utilization, machine reconfigurability and operational capability by applying the Nondominated Sorting Genetic Algorithm II (NSGA II).

\section{Performance Measures}

In a reconfigurable manufacturing system, the machines are capable of performing variety of operations in its existing configurations and the reconfigurable machine tools (RMT) can further be reconfigured into other configurations. The different configurations thus can further enhance the functionality and can perform number of operations. In such a scenario the availability of large number of machines 
to perform a single operation, makes it a complex problem to assign the RMTs to the reconfigurable flow lines. Thus to access the suitability of a configuration effectively the performance indicators like machine reconfigurability, operational capability, machine utilization along with the cost are considered in this chapter to optimize the reconfigurable flow lines.

\section{Notations:}

$m c_{i}^{j} \quad$ machine $i(1<i<I)$ in its $j^{\text {th }}\left(1<j<J_{i}\right)$ configuration

$n_{i}^{j} \quad$ number of machines required to satisfy the demand when machine $i$ with $j^{\text {th }}$ configuration is selected

$D$ demand rate

$F S_{\mathrm{k}} \quad$ a set of feasible alternative machine configurations to perform $k^{\text {th }}(1<k<K)$ operation $\left\{\left(i_{1}, j_{1}\right),\left(i_{2}, j_{2}\right), \ldots .\left(i_{f} j_{f}\right) \ldots \ldots .\left(i_{F_{k}}, j_{F_{k}}\right)\right\}$. Here each feasible alternative $f\left(1<f<F_{k}\right)$ is defined as $\left(i_{f}, j_{f}\right)$, where $i_{f}$ specifies the feasible machine and $j_{f}$ specifies the feasible machine configuration

$C M_{i}^{j} \quad$ cost of machine $i$ with $j^{\text {th }}$ configuration (i.e. $m c_{i}^{j}$ )

$P^{j} \quad$ production rate of machine $i$ with $j^{\text {th }}$ configuration for performing $k^{\text {th }}$ $i, k \quad$ operation

$\delta^{j} \quad 1$ if operation $k$ can be performed with machine $i$ having its $j^{\text {th }}$ configuration, $\delta_{i, k} \quad$ otherwise 0

$C_{p, q} \quad$ cost of assigning $p^{\text {th }}$ machine with $q^{\text {th }}$ configuration from the feasible alternative machine configurations to perform an operation at specified demand rate

$M U_{p, q}$ machine utilization of assigning $p^{\text {th }}$ machine with $q^{\text {th }}$ configuration from the feasible alternative machine configurations to perform an operation at specified demand rate

$C C_{p, q}$ configuration convertibility of assigning $p^{\text {th }}$ machine with $q^{\text {th }}$ configuration from the feasible alternative machine configurations to perform an operation at specified demand rate

$O C_{p, q}$ operational capability of assigning $p^{\text {th }}$ machine with $q^{\text {th }}$ configuration from the feasible alternative machine configurations to perform an operation at specified demand rate

$M R_{p, q}$ Machine reconfigurability of assigning $p^{\text {th }}$ machine with $q^{\text {th }}$ configuration from the feasible alternative machine configurations to perform an operation at specified demand rate

\section{$2.1 \operatorname{Cost}(C)$}

Cost is the important performance parameter driving the selection of machine configuration for a particular operation. Thus meeting the customer demands economically is most important. The cost $\left(C_{p, q}\right)$ of a feasible alternative machine configuration for performing $k^{\text {th }}$ operation at specified demand rate is calculated using: 


$$
\begin{aligned}
C_{p, q} & =n_{p}^{q} \times C M_{p}^{q} \\
\text { where } \quad n_{p}^{q} & =\left\lceil\frac{D}{P_{p, k}^{q}}\right\rceil
\end{aligned}
$$

\subsection{Machine Utilization (MU)}

In the present scenario industries are facing a stiff global competition and volatile markets. In such circumstances utilization of the manufacturing system capacity is very crucial for sustenance and growth of the concern and underutilization of machine capacity which in turn affects the economic functioning may pose a serious threat to the survival of industry. Therefore the system should be utilized to the maximum possible extent by optimizing the system configuration. In the reconfigurable manufacturing environment the availability of multifunctional machines which can further be reconfigured into various configurations turns the machine selection problem into combinatorial problem. Therefore, while selecting the system configuration for a part the machine utilization should be given due consideration.

$$
M U_{p, q}=\frac{D}{P_{p, k}^{q} \times n_{p}^{q}}
$$

\subsection{Operational Capability (OC)}

The capability of an RMT to readily perform a variety of operations in its existing configuration gives an upper edge to respond to the dynamic behavior of the market and turns into high responsiveness. For performing a particular operation $k$ $(1<k<K)$ the operational capability of a feasible alternative machine configuration is computed based upon the variety of operations that can be performed by the machine in its present configuration. As the number of operations that can be performed by a machine increases, its contribution to the operational capability also increases. Our objective is to maximize the operational capability, therefore the operational capability contribution of every additional increase in the operations performed must reflect more pronounced value of operational capability as compared to that of lower values of operational capability. To reflect this consideration a power index $\mathrm{Y}$ is used. Deciding the value of $\mathrm{Y}$ is a matter of sensitivity analysis which may be carried out to see the overall impact of value of $\mathrm{Y}$ on machine selection and its impact on the reconfiguration efforts over the entire planning horizon. The operational capability $\left(O_{p, q}\right)$ of a feasible alternative machine configurations to perform a particular operation is computed using:

$$
O C_{p, q}=\left[\left(\sum_{k=1}^{K} \delta_{p, k}^{q}\right)-1\right]^{Y}
$$




\subsection{Machine Reconfigurability (MR)}

The quick adaptability of the reconfigurable manufacturing system in response to the dynamic environment is achieved by reconfiguration of the machines. Thus reconfigurability is a criterion to judge the adaptability of the machine configuration. In the present work a novel approach to measure the reconfigurability of an RMT is proposed based on the number of configurations into which an existing machine configuration may be converted along with considering the effort required in conversions in the form of adding/removing and/or readjusting the auxiliary modules. The effort in each configuration conversion is being calculated by a methodology based on set theory. As shown in Fig. 3 in each conversion two sets of auxiliary modules are participating one is the set of auxiliary modules of existing machine configuration and the other is the set of auxiliary modules required in the new configuration. Thus the total auxiliary modules i.e. union of the both sets of auxiliary modules is categorized into three classes, the auxiliary modules to be added, removed and readjusted. Here it is assumed that the existing modules which are retained in the next configuration need to be readjusted. Further the ratio of three classes of auxiliary modules to the total modules is multiplied by the weights $\alpha, \beta$ and $\gamma$ which gives the effort required in machine configuration conversion. In this way the total effort required for all the possible conversions of existing configuration is calculated. The total reconfiguration effort required is also dependant on the number of copies of the machine configuration required to satisfy the demand rate with the existing configuration, as all the machines are to be reconfigured to change the configuration of existing machine configuration. Therefore the earlier computed effort is multiplied by the number of machines calculated through Eq. (2).

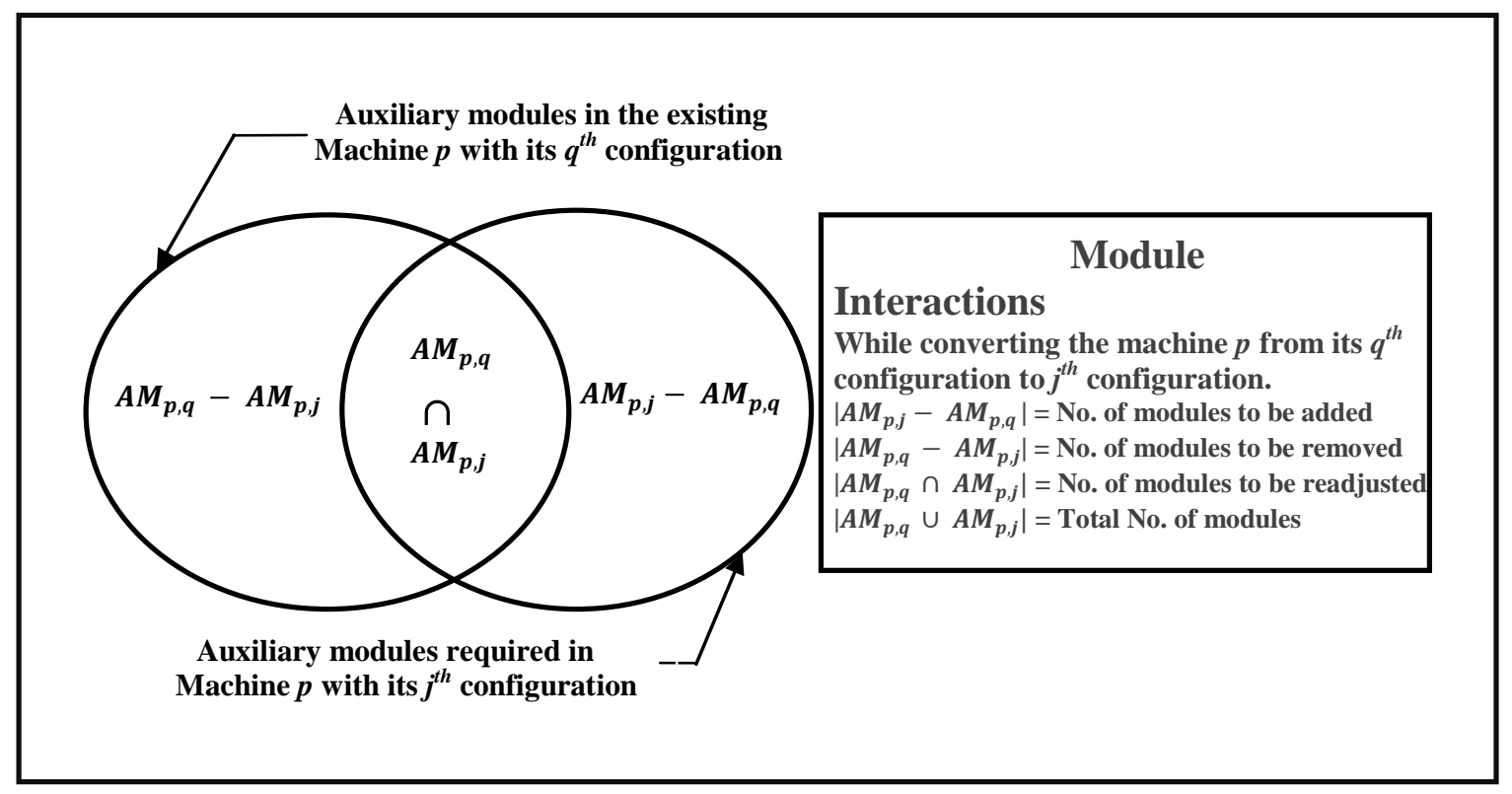

Fig. 3. Reconfigurability effort calculation through set theory

For computing the reconfigurability of a machine configuration, the number of configurations into which it can be converted plays a vital role, if a machine is having only one configuration $\left(\mathrm{J}_{\mathrm{p}}=1\right)$ i.e. it cannot be converted into further any configurations, thus it will not make any contribution in reconfigurability. As the 
number of configurations into which a machine can be converted increases, its contribution to the reconfigurability also increases. As the objective in general is to maximize the reconfigurability, therefore every additional increase in the number of possible configurations must reflect an increased value of reconfigurability. Therefore the reconfigurability contribution of every additional increase in the $J_{p}$ must reflect more pronounced value of reconfigurability as compared to that of $\mathbf{J}_{\mathrm{p}}-1$. To reflect this consideration a power index $\mathrm{z}$ is used in the Eq. (5). Therefore the reconfigurability $\left(R_{p, q}\right)$ of a machine configuration is calculated using the following equation.

$$
\begin{aligned}
R_{p, q}=\left[J_{p}-1\right]^{Z} / n_{p}^{q} \sum_{j=1, j \neq q}^{J_{p}}\left[\begin{array}{c}
\alpha \frac{\text { No.of modules added }}{\text { Total modules }}+\beta \frac{\text { No. of modules removed }}{\text { Total modules }} \\
+\gamma \frac{\text { No.of modules readjusted }}{\text { Total modules }}
\end{array}\right] \\
=\frac{\left[\mathrm{J}_{\mathrm{p}}-1\right]^{\mathrm{z}}}{\mathrm{n}_{\mathrm{i}}^{\mathrm{j}} \sum_{\mathrm{j}=1, \mathrm{j} \neq \mathrm{q}}^{\mathrm{J}_{\mathrm{p}}}\left[\alpha \frac{\mathrm{AM}_{\mathrm{p}, \mathrm{j}}-\mathrm{AM}_{\mathrm{p}, \mathrm{q}}}{\mathrm{AM}_{\mathrm{p}, \mathrm{q}} \cup \mathrm{AM}_{\mathrm{p}, \mathrm{j}}}+\beta \frac{\mathrm{AM}_{\mathrm{p}, \mathrm{q}}-\mathrm{AM}_{\mathrm{p}, \mathrm{j}}}{\mathrm{AM}_{\mathrm{p}, \mathrm{q}} \cup \mathrm{AM}_{\mathrm{p}, \mathrm{j}}}+\gamma \frac{\mathrm{AM}_{\mathrm{p}, \mathrm{q}} \cap \mathrm{AM}_{\mathrm{p}, \mathrm{j}}}{\mathrm{AM}_{\mathrm{p}, \mathrm{q}} \cup \mathrm{AM}_{\mathrm{p}, \mathrm{j}}}\right]}
\end{aligned}
$$

Generally $\alpha>\beta>\gamma$, as the effort required in adding the module is comparatively higher than removing the module and further the effort required in removing the module is reasonably higher than just readjusting the existing modules.

\section{Designing Optimal Reconfigurable Flow Line using NSGA II}

Deb et al. (2002) proposed NSGA-II, which is one of the most efficient and famous multi-objective evolutionary algorithms. The algorithm applies the fast nondominated sorting technique and a crowding distance to rank and select the population fronts. The terminologies central to the concept (non dominated sorting procedure, crowded distance estimation, crowded comparison operator) of NSGA-II, may be referred from Deb et al. (2002). The performance indices discussed above are applied to optimize reconfigurable flow line allowing paralleling of similar machines. As shown in Fig. 4, each operation is assigned to a stage according to the precedence constraints of the operation sequence and each stage is further assigned a machine type and its configuration number. The optimal assignment of the machine and machine configurations is realized by NSGA II taking cost, machine utilization, operational capability and machine reconfigurability as the objectives. For applying NSGA II in the present study, the set of feasible alternative machine configurations $\left(F S_{k}\right)$ for all the operations are recorded beforehand. Each element $f$ of set $F S_{k}$ is a combinations of two parameters i.e. machine and the machine configuration. Total number of feasible alternate machine configurations to perform the $k^{\text {th }}$ operation is $F_{k}$. The recording of feasible alternative machine configurations for all the operations is necessary for the constraint handling by applying the real coded chromosomes. 


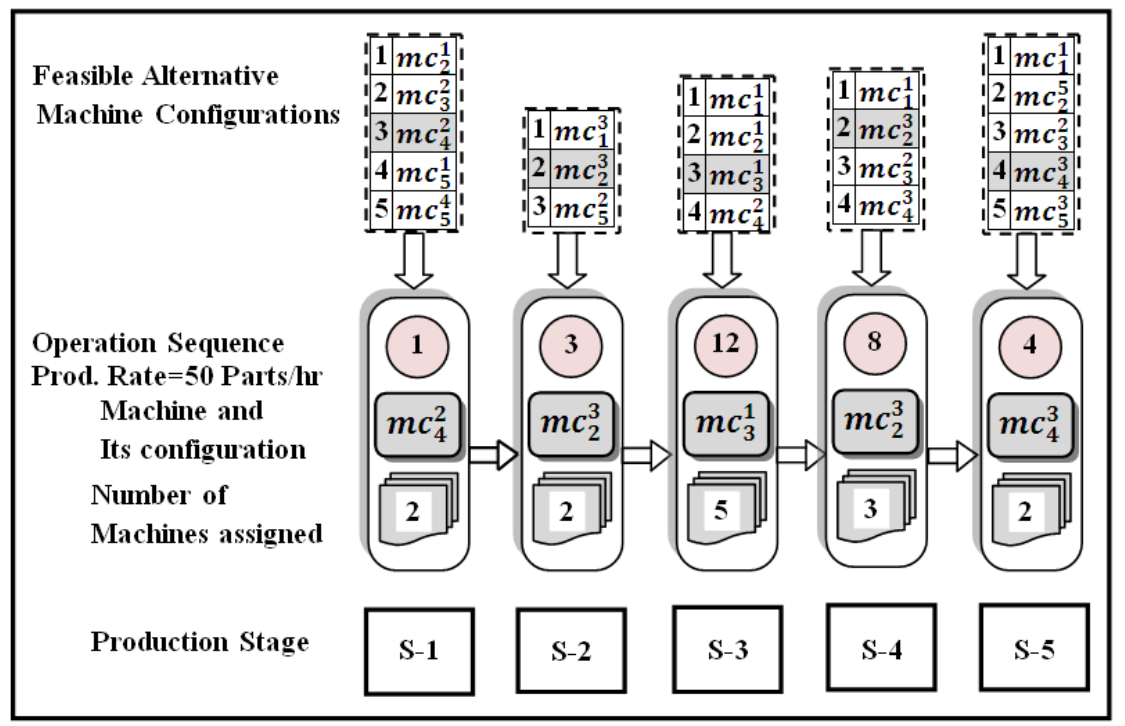

Fig. 4. RMS Flow line feasible machine configurations mapping

\section{Objective Function, Constraint Handling and Solution Mapping}

The present study proposes the assignment of machines to all the operations allocated on production stages from the feasible alternative machine configurations based on the objective function:

$$
\begin{aligned}
& \text { Minimize } F 1=\sum_{s=1}^{S} C_{p_{s}, q_{s}} \\
& \text { Maximize } F 2=\sum_{s=1}^{S} M U_{p_{s}, q_{s}} \\
& \text { Maximize } F 3=\sum_{s=1}^{S} O C_{p_{s}, q_{s}} \\
& \text { Maximize } F 4=\sum_{s=1}^{S} M R_{p_{s}, q_{s}}
\end{aligned}
$$

Here Equations (6) to (9) represent the objectives cost, machine utilization, operational capability and machine reconfigurability respectively. The subscripts $p_{s}$ and $q_{s}$ represent the feasible alternative machine $p$ with its configuration $q$ assigned at the $s^{\text {th }}$ stage. The real-encoding of chromosome is used to overcome the difficulties related to binary encoding of continuous parameter optimization problems (Goldberg, 1991; Wright, 1991; Michalewicz, 1992). The real coded chromosomes along with the NSGA II are employed in the present study for obtaining the nondominated solutions representing optimal machine assignment. But in the present case feasible solutions are rather sparse which will lead to infeasible population. Further the crossover and mutation of the chromosomes will give rise to the infeasibility. Therefore a real coded chromosome is proposed. The length of chromosome is equal to the number of stages and on each stage an operation has to be performed according to the operation sequence. A set of feasible alternative machine configurations $F S_{k}$ for each operation is already recorded as shown in Fig. 4. As shown in Fig. 5, now each 
stage is to be assigned with a feasible machine configuration which is mapped in the present study through the real coded chromosomes.

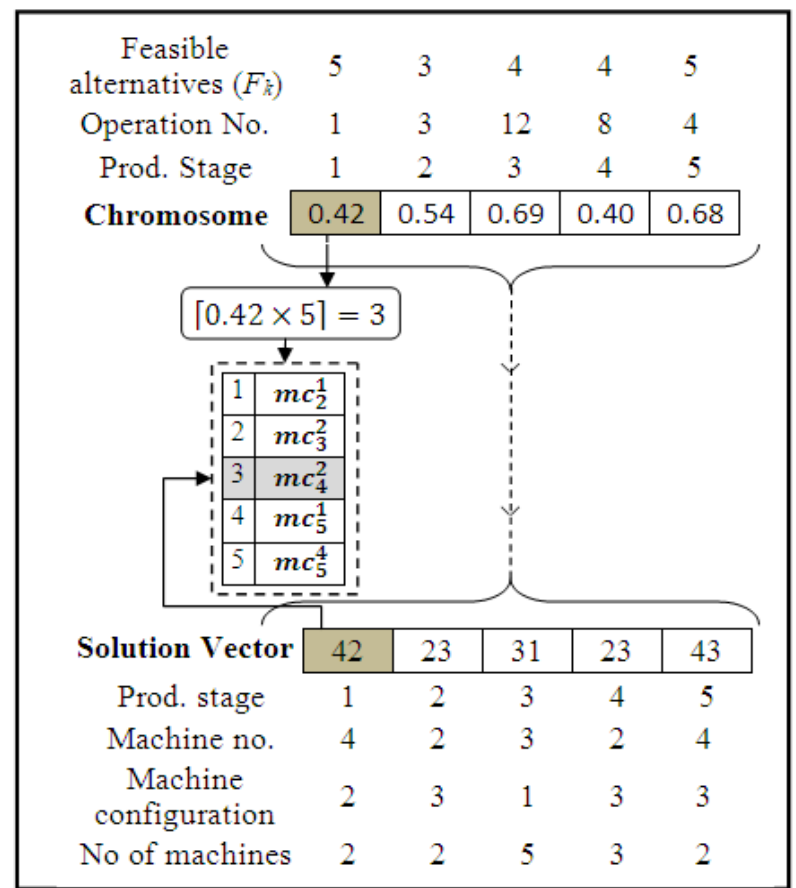

Fig. 5. Real coded chromosome solution mapping

\section{Case Study}

For illustrating the developed approach of optimally designing the reconfigurable flow line, a set of RMTs given in Tab. 1 having the operational capabilities as given in Tab. 2 is considered. The optimal configuration selection for the reconfigurable flow line allowing paralleling of similar machines for a single fixed demand period is illustrated. As shown in Figure 5, the operation sequence of the part to be produced is assumed to be $1 \rightarrow 3 \rightarrow 12 \rightarrow 8 \rightarrow 4$ with a demand rate of 50 . The number of stages is also assumed to be five. The default parameters chosen in the present illustration are $\alpha, \beta, \gamma$ as $0.5,0.4,0.1$ respectively and the value of power indices $\mathrm{Y}$ and $\mathrm{Z}$ in the Eq. (4) and (5) are assumed as 2. Fig. 4. represents one of the 63 non dominated solutions for the considered reconfigurable flow line obtained by applying NSGA II to the present case study and Fig. 5 represents the solution mapping of the real coded chromosome for $2^{\text {nd }}$ production stage of the reconfigurable flow line. The sample computation of performance measures is illustrated considering the first production stage.

\subsection{Performance Indices Computation Illustration}

(i) Cost: The second production stage shown in Fig. 4, performs operation number 3 and the machine configuration assigned is $m c_{2}^{3}$, According to Eq. 2 the number of machines required are 2 and the cost may be computed using Eq. 1 as $2 \times 1140=2280 \times 10^{3}$ US\$. The cost of the whole flow line would be the sum of the all stages which is $15400 \times 10^{3}$ US\$. 
(ii) Machine utilization: The required production rate is $50 \mathrm{parts} / \mathrm{hr}$ and for the selected machine configuration $m c_{2}^{3}$ on production stage 2 . The production rate of selected machine configuration is $25 \mathrm{parts} / \mathrm{hr}$, therefore machine utilization accordingto Eq. (3) is 1.0. The machine utilization of the whole flow line would be the average of the all stages which is 0.90 in this case.

\begin{tabular}{|c|c|c|c|c|}
\hline Machine & Machine Configuration & Basic Modules & Auxiliary Modules & $\begin{array}{c}\text { Cost } \\
\text { (in } 10^{3} \text { of USD) }\end{array}$ \\
\hline \multirow{3}{*}{$M_{1}$} & $m c_{1}^{1}$ & $\{01,05\}$ & $\{13,17,21,22\}$ & 750 \\
\cline { 2 - 5 } & $m c_{1}^{2}$ & $\{01,05\}$ & $\{12,13,15,20,21\}$ & 955 \\
\cline { 2 - 5 } & $m c_{1}^{3}$ & $\{01,05\}$ & $\{11,17,18,20,21\}$ & 1025 \\
\hline \multirow{3}{*}{$M_{2}$} & $m c_{1}^{4}$ & $\{01,05\}$ & $\{15,17,18\}$ & 840 \\
\cline { 2 - 5 } & $m c_{2}^{1}$ & $\{02,04,08\}$ & $\{11,13,16,22,24\}$ & 1215 \\
\cline { 2 - 5 } & $m c_{2}^{2}$ & $\{02,04,08\}$ & $\{14,16,19\}$ & 910 \\
\cline { 2 - 5 } & $m c_{2}^{3}$ & $\{02,04,08\}$ & $\{13,19,24\}$ & 1140 \\
\hline \multirow{3}{*}{$M_{3}$} & $m c_{2}^{4}$ & $\{02,04,08\}$ & $\{11,13,15,18,24\}$ & 1350 \\
\cline { 2 - 5 } & $m c_{2}^{5}$ & $\{03,05,07\}$ & $\{11,12,14,18\}$ & 1050 \\
\hline \multirow{3}{*}{$M_{4}$} & $m c_{3}^{1}$ & $\{03,05,07\}$ & $\{12,13,14,17,19,19\}$ & 780 \\
\cline { 2 - 5 } & $m c_{3}^{2}$ & $\{04,09\}$ & $\{18,23\}$ & 1825 \\
\cline { 2 - 5 } & $m c_{4}^{1}$ & $\{04,09\}$ & $\{11,15,18,20,21\}$ & 1350 \\
\hline \multirow{3}{*}{$M_{5}$} & $m c_{4}^{2}$ & $\{03,06,10\}$ & $\{13,14,17,18\}$ & 1400 \\
\cline { 2 - 5 } & $m c_{4}^{3}$ & $\{03,06,10\}$ & $\{16,17,19,20,25\}$ & 1175 \\
\cline { 2 - 5 } & $m c_{5}^{1}$ & $\{03,06,10\}$ & $\{11,12,13,15,22\}$ & 1230 \\
\cline { 2 - 5 } & $m c_{5}^{2}$ & $\{03,06,10\}$ & $\{20,22,24\}$ & 1175 \\
\hline
\end{tabular}

Tab. 1. RMT module requirements and cost

\begin{tabular}{|c|c|c|c|c|c|c|c|c|c|c|c|c|c|c|c|c|c|c|c|c|}
\hline \multirow{2}{*}{$\begin{array}{l}\text { Operation } \\
(\mathrm{k}) \rightarrow \\
m c_{i}^{j} \downarrow\end{array}$} & \multicolumn{20}{|c|}{ RMT Production rate in parts/hour for performing various operations } \\
\hline & 1 & 2 & 3 & 4 & 5 & 6 & 7 & 8 & 9 & 10 & 11 & 12 & 13 & 14 & 15 & 16 & 17 & 18 & 19 & 20 \\
\hline$m c_{1}^{1}$ & - & - & - & 14 & - & - & - & 12 & - & - & - & 8 & - & - & - & 18 & - & - & - & - \\
\hline$m c_{1}^{2}$ & - & - & - & - & 15 & - & - & - & 20 & - & - & - & - & - & - & - & - & 16 & - & - \\
\hline$m c_{1}^{3}$ & - & - & 20 & - & - & - & 15 & - & - & - & - & - & - & - & - & 25 & - & - & - & - \\
\hline$m c_{1}^{4}$ & - & - & - & - & - & - & - & - & - & 15 & - & - & - & - & - & - & - & - & 12 & - \\
\hline$m c_{2}^{1}$ & 14 & - & - & - & - & 15 & - & - & - & - & - & 12 & - & - & - & - & - & - & - & 20 \\
\hline$m c_{2}^{2}$ & - & 15 & - & - & - & - & - & - & - & - & - & - & 14 & - & 15 & - & - & - & - & - \\
\hline$m c_{2}^{3}$ & - & - & 25 & - & - & - & - & 18 & - & - & 25 & - & - & - & - & - & 20 & - & - & - \\
\hline$m c_{2}^{4}$ & - & 20 & - & - & 20 & - & 18 & - & - & - & - & - & - & 24 & - & - & - & - & - & - \\
\hline$m c_{2}^{5}$ & - & - & - & 18 & - & - & - & - & - & - & - & - & 20 & - & - & - & - & 14 & - & 15 \\
\hline$m c_{3}^{1}$ & - & 12 & - & - & - & - & - & - & 15 & - & - & 10 & - & - & - & - & 10 & - & - & - \\
\hline$m c_{3}^{2}$ & 30 & - & - & 26 & - & - & - & 24 & - & - & 24 & - & - & - & 20 & - & 35 & - & 15 & - \\
\hline$m c_{4}^{1}$ & - & - & - & - & - & 25 & - & - & - & 30 & - & - & - & - & - & - & - & 25 & - & - \\
\hline$m c_{4}^{2}$ & 25 & - & - & - & - & - & - & - & - & - & - & 22 & - & - & - & - & 30 & - & - & 26 \\
\hline$m c_{4}^{3}$ & - & 18 & - & 25 & - & - & - & 16 & - & - & - & - & 22 & - & - & 28 & - & - & 20 & - \\
\hline$m c_{5}^{1}$ & 16 & - & - & - & - & - & 15 & - & - & - & 15 & - & - & 18 & - & - & - & 18 & - & - \\
\hline$m c_{5}^{2}$ & - & - & 24 & - & 20 & - & - & - & - & 25 & - & - & - & - & - & - & 24 & - & - & 20 \\
\hline$m c_{5}^{3}$ & - & - & - & 24 & - & - & - & - & 30 & - & - & - & - & - & 18 & - & - & - & - & - \\
\hline$m c_{5}^{4}$ & 20 & - & - & - & - & 22 & 14 & - & - & - & - & - & - & 20 & - & 16 & - & - & 18 & - \\
\hline
\end{tabular}

Tab.2. Operational capabilities of RMTs 
(iii) Operational capability: The operational capability is calculated for the $m c_{2}^{3}$ using Eq. (4) as an illustration. The machine $m c_{2}^{3}$ is capable of performing operations $3,8,11$, and 17 . Thus the operation capability for $m c_{2}^{3}$ is $[(4)-1]^{2}=9$. The operational capability of the whole flow line would be the sum of all production stages which is 61.

(iv) Machine reconfigurability: The reconfigurability of machine configuration $m c_{2}^{3} \quad(p=2, q=4)$ is computed for an illustration. The number of possible configurations $J_{p}$ into which machine number 2 can be converted is 5 (see Table 1), thus the machine $m c_{2}^{4}$ can be further converted into 4 more configurations i.e. $\left[J_{p}-1\right]$ is $[5-1]=4$ in this case. The effort in each of these conversions is computed and summed up in the denominator in Equation (4). We illustrate the effort involved in the conversion of present machine configuration $m c_{2}^{3}$ into $m c_{2}^{1}$. As shown in Fig. 3, during this conversion the number of modules to be added ( $\mid A M_{2,1}-$ $\left.A M_{2,3} \mid\right)$ are 3 i.e. $\{11,16,22\}$, number of modules to be removed $\left(\mid A M_{2,3}-\right.$ $\left.A M_{2,1} \mid\right)$ is 1 i.e. $\{19\}$ and number of modules to be readjusted $\left(\left|A M_{2,3} \cap A M_{2,1}\right|\right)$ are 2 i.e. $\{13,24\}$. The total number of modules $\left(\left|A M_{2,4} \cup A M_{2,1}\right|\right)$ in this conversion are 6 i.e. $\{11,13,16,19,22,24\}$. Thus effort required in this conversion is computed as $[(0.5 * 3 / 6)+(0.4 * 1 / 6)+(0.1 * 2 / 6)]$ which is 0.35 . Similarly the effort required in other three conversions $\left(m c_{2}^{2}, m c_{2}^{4}, m c_{2}^{5}\right)$ is $0.38,0.35,0.45$. Thus total reconfiguration effort in all the four possible conversions is $0.35+0.38+0.35+0.45=$ 1.53. The number of machines required in this case is two, thereby the total effort involved in all the two machine conversions (denominator of Eq. (5)) is $2 * 1.53=3.06$ and the machine reconfigurability $\left(R_{p, q}\right)$ of $m c_{2}^{4}$ is $\left[(5-1)^{2} / 3.06\right]=5.23$. The machine reconfigurability of the whole flow line would be the sum of all stages which is 14.44 in this case.

\subsection{Nondominated Solutions from NSGA II}

The non dominated sorting algorithm II has been applied to optimally design the configuration of the reconfigurable flow line considered in the present case study. The various parameters used in NSGA II are Population size 50, Number of generations 100 , binary tournament selection operator, crossover probability 0.8 and mutation probability as 0.2 . As the present problem lies in the domain of discrete discontinuous and non convex search space, thus predicting the exact number of pareto frontiers is not possible. The non dominated solutions obtained in the present study are 63. The best three solutions in respect of each objective i.e. cost, machine utilization, operational capability and machine reconfigurability are presented in the Tab. 3. The selected machine configurations and the number of copies of the machine configuration required are also listed. 


\begin{tabular}{|c|c|c|c|c|c|c|c|c|c|c|}
\hline $\begin{array}{l}\text { S. } \\
\text { No. }\end{array}$ & \multicolumn{4}{|l|}{$\begin{array}{l}\text { Solutions (Stage wise) } \\
\text { M/c config. assigned/No. of m/c }\end{array}$} & $\begin{array}{l}\text { Cost } \\
(C)\end{array}$ & $\begin{array}{l}\text { Op. } \\
\text { Cap. }(O C)\end{array}$ & $\begin{array}{l}\text { M/c } \\
\text { Rec. }(M R)\end{array}$ & $\begin{array}{l}\text { M/c } \\
\text { Util. }(M U)\end{array}$ & $\begin{array}{l}\text { Sol. } \\
\text { No. }\end{array}$ \\
\hline 1 & $42 / 2$ & $23 / 2$ & $31 / 5$ & $23 / 3$ & $43 / 2$ & 15400 & 61.00 & 14.44 & 0.99 & 51 \\
\hline 2 & $42 / 2$ & $23 / 2$ & $31 / 5$ & $23 / 3$ & $25 / 3$ & 15750 & 45.00 & 15.20 & 0.97 & 57 \\
\hline 3 & $54 / 3$ & $23 / 2$ & $31 / 5$ & $23 / 3$ & $43 / 2$ & 15925 & 77.00 & 14.75 & 0.95 & 4 \\
\hline 4 & $32 / 2$ & $52 / 3$ & $31 / 5$ & $32 / 3$ & $32 / 2$ & 20200 & 133.00 & 6.66 & 0.84 & 54 \\
\hline 5 & $32 / 2$ & $52 / 3$ & $42 / 3$ & $32 / 3$ & $32 / 2$ & 20800 & 133.00 & 7.87 & 0.79 & 61 \\
\hline 6 & $32 / 2$ & $52 / 3$ & $11 / 7$ & $32 / 3$ & $32 / 2$ & 21550 & 133.00 & 7.30 & 0.82 & 32 \\
\hline 7 & $54 / 3$ & $23 / 2$ & $21 / 5$ & $23 / 3$ & $25 / 3$ & 18450 & 61.00 & 17.28 & 0.90 & 46 \\
\hline 8 & $21 / 4$ & $23 / 2$ & $21 / 5$ & $23 / 3$ & $25 / 3$ & 19785 & 45.00 & 17.25 & 0.92 & 41 \\
\hline 9 & $42 / 2$ & $23 / 2$ & $21 / 5$ & $23 / 3$ & $25 / 3$ & 17925 & 45.00 & 16.97 & 0.94 & 16 \\
\hline 10 & $42 / 2$ & $23 / 2$ & $31 / 5$ & $23 / 3$ & $43 / 2$ & 15400 & 61.00 & 14.44 & 0.99 & 51 \\
\hline 11 & $42 / 2$ & $23 / 2$ & $31 / 5$ & $23 / 3$ & $32 / 2$ & 16250 & 72.00 & 13.22 & 0.98 & 36 \\
\hline 12 & $42 / 2$ & $23 / 2$ & $31 / 5$ & $23 / 3$ & $25 / 3$ & 15750 & 45.00 & 15.20 & 0.97 & 57 \\
\hline
\end{tabular}

Tab. 3. Non-dominated solutions for the considered reconfigurable flow line

\section{Summary and Further Research}

This chapter described an approach to optimally design the reconfigurable flow lines. In the present study a novel approach has been proposed for the machine selection based on machine utilization, operational capability, machine reconfigurability along with the cost. The multiple objective problem of machine assignment is attempted through NSGA II. The present problem lies in the domain of discrete and discontinuous search space and the developed approach of applying the real coded chromosomes helped in handling the sparse population of feasible solutions along with facilitating the crossover and mutation. Form the list of non dominated solutions depicted in the Tab. 3. It can be seen that the higher values of operational capability and the machine reconfigurability turn into the associated higher costs initially but in future the saving into the required reconfiguration efforts would certainly surpass the initially excessive costs incurred. The decision manager may choose a suitable candidate from the nondominated solutions to justify the management policy and the market trends. In future the RMS configuration may be designed for multiple product reconfigurable flow lines. The alternative routing of parts may also be considered in the future research works.

\section{References}

Dou, J.; Dai, X. \& Meng, Z. (2009). Graph theory-based approach to optimise single product flow-line configuration of RMS. International Journal of Advanced Manufacturing Technology, Vol. 41, No. 9-10, pp. 916-931

Goyal, K. K.; Jain, P. K. \& Jain, M. (2011a). A Novel Approach to Measure Machine Reconfigurability in Reconfigurable Manufacturing System, Annals of DAAAM for 2011 \& Proceedings of the 22nd International DAAAM Symposium, Editor B[ranko] Katalinic, ISBN 978-3-901509-83-4, ISSN 1726-9679, pp 09590960, Published by DAAAM International, Vienna, Austria 2011 
Goyal, K. K.; Jain, P. K. \& Jain, M. (2011b). Optimal configuration selection for reconfigurable manufacturing system using NSGA II and TOPSIS. International Journal of Production Research, (iFirst) DOI:10.1080/00207543.2011.599345

Gumasta, K.; Gupta, S. K.; Benyoucef, L. \& Tiwari, M. K. (2011). Developing a reconfigurability index using multi-attribute utility theory. International Journal of Production Research, Vol., 49 No., 6, pp. 1669 - 1683

Koren, Y.; Hiesel, U.; Jovane, F.; Moriwaki, T.; Pritschow, G.; Ulsoy, G. \& Van, B. H. (1999). Reconfigurable Manufacturing Systems. Annals of the CIRP, Vol. 48, No. 2, pp. 527-540

Liles, D. H. \& Huff, B. L. (1990). A computer based computer scheduling architecture suitable for driving a reconfigurable manufacturing system. Computers and Industrial Engineering, Vol. 19, No. 1-4, pp. 1-5

Landers, R. G. A. (2000). New paradigm in machine tools: Reconfigurable machine tools, Japan-USA Symposium on Flexible Automation, Ann Arbor, Michigan

Mehrabi, M. G.; Ulsoy, A. G. \& Koren, Y. (2000). Reconfigurable manufacturing systems: Key to future manufacturing. Journal of Intelligent Manufacturing, Vol. 11, No. 4, pp. 403-419

Michalewicz, Z. (1992). Genetic algorithms + data structures $=$ evolution programs. New York: Springer-Verlag

Moon, Y. M. \& Kota, S. (2002). Design of reconfigurable machine tools. Journal of Manufacturing Science and Engineering, Vol. 124, No. 2, pp. 480-83

Pattanaik, L. N.; Jain, P. K. \& Mehta, N. K. (2007). Cell formation in the presence of reconfigurable machines, International Journal of Advanced Manufacturing Technology, Vol. 34, No. 2, pp. 335-345

Son, S. Y.; Olsen, T. L. \& Derek, Y. (2001). An approach to scalability and line balancing for RMS. Integrated Manufacturing Systems, Vol. 12, No. 7, pp. 500-511.

Spicer, P. \& Carlo, H. J. (2007). Integrating reconfiguration cost into the design of multi-period scalable reconfigurable manufacturing systems, Transactions of the ASME, Vol. 129, pp. 202-210

Tsukune. H.; Tsukamoto, M.; Matsushita, T.; Tomita, F.; Okada, K.; Ogasawara, T.; Takase, K. \& Tuba, T. (1993) Modular manufacturing. Journal of intelligent manufacturing, Vol. 4, No. 2, pp. 163-81

Wright, A. H. (1991). Genetic algorithms for real parameter optimization, In: Foundations of Genetic Algorithms. G.J.E. Rawlins, ed., pp. 205-218, San Mateo: Morgan Kaufmann

Youssef, A. M. A. \& El Maraghy, H. A. (2006). Modelling and optimisation of multiple-aspect RMS configuration. International Journal of Production Research, Vol. 44, No. 22, pp. 4929-4958

Youssef, A. M. A. \& ElMaraghy, H. A. (2007). Optimal configuration selection for reconfigurable manufacturing systems. International Journal of Flexible Manufacturing Systems, Vol. 19, No. 2, pp. 67-106 